Pacific Journal of Mathematics

PERMANENT INEQUALITY FOR POSITIVE FUNCTIONS ON 


\title{
A PERMANENT INEQUALITY FOR POSITIVE FUNCTIONS ON THE UNIT SQUARE
}

\author{
Morton L. Slater and ROBERT J. ThOMPSON
}

Introduction. During the past few years the van der Waerden conjecture on the minimum of the permanent of a doubly stochastic matrix has received considerable attention. (See Marcus and Newman [1] and [2], Marcus and Minc [1], among others.) This conjecture states that if $A$ is a doubly stochastic matrix, i.e. if

$$
a_{i j} \geqq 0, \sum_{i=1}^{n} a_{i j}=\sum_{j=1}^{n} a_{i j}=1,
$$

then the permanent of $A$ is $\geqq n ! n^{-n}$. (The permanent of $A$ is $\sum \prod a_{i \sigma(i)}$, where the summation is taken over all permutations $\sigma$ in the symmetric group.) Despite the seemingly elementary character of the conjecture, it is, so far as the present authors are aware, still unresolved in general, although it has been settled in some special cases. (See the above references.)

An implication of the conjecture is that some term of the permanent expansion must be greater than or equal to $n^{-n}$. This was established by Marcus and Minc [1] in 1962. Specifically they showed that if $\Pi a_{i i}$ is not exceeded by any other term in the permanent expansion, then

$$
\sum \log a_{i i} \geqq \sum \sum a_{i j} \log a_{i j} \geqq \mathrm{n} \log n^{-1} .
$$

The second inequality above is a simple application of Jensen's inequality using the convex function $x \log x$; the first inequality is the key to the problem. It is the extension of this inequality to functions defined on the unit square that is referred to in the title of this paper. We will show in $\S 4$ that under suitable hypotheses

$$
\infty>\int_{0}^{1} \log f(x, x) d x \geqq \int_{0}^{1} \int_{0}^{1} f(x, y) \log f(x, y) d x d y \geqq 0 .
$$

The proof of (2) (and incidentally a new proof of (1)) is based ultimately on the following theorem:

THEOREM 1. Let $S$ be an arbitrary set and $f(p, q)$ a real-valued function defined on $S \times S$ with the following property:

(C) if $p_{1}, \cdots, p_{n}$ is any finite sequence of points in $S$, not necessarily distinct, then

Received September 12, 1963. This work was performed under the auspices of the United States Atomic Energy Commission. 


$$
f\left(p_{1}, p_{2}\right)+f\left(p_{2}, p_{3}\right)+\cdots+f\left(p_{n-1}, p_{n}\right)+f\left(p_{n}, p_{1}\right) \leqq 0 .
$$

Then there exists a real valued function $\varphi$ defined on $S$ such that for all $(p, q) \in S \times S$

$$
f(p, q) \leqq \varphi(p)-\varphi(q) .
$$

Furthermore, given any $s \in S$, we may determine $\varphi(p)$ so that for all $p \in S$

$$
f(p, s) \leqq \varphi(p) \leqq-f(s, p), \quad \text { and } \varphi(s)=0 \text {. }
$$

This theorem for finite sets $S$ is essentially contained in a paper by S. N. Afriat [1] which appeared in 1963 in connection with a study of empirical preference analysis in economics. Theorem 1 was discovered independently by the authors in their study of the van der Waerden conjecture; it is very closely related to the linear programming dual of a theorem proved by Garret Birkhoff [1], which states that the doubly stochastic matrices are the convex hull of the permutation matrices. Indeed it was this last fact which persuaded us that Theorem 1 could be applied directly to the van der Waerden conjecture. In $\S 1$ we will give a proof of this theorem which differs essentially from that for the finite case given by Afriat; it is certainly much shorter.

The proof of (2) to be given in $\$ \S 3$ and 4 will depend on Theorem 1 and on the following "Arzela type" compactness result proved by M. Riesz. We state it, for reference, in the form that we shall use it. It is also convenient to state here a partial converse of the Fubini theorem proved by L. Tonelli.

THEOREM A (M. Riesz). Let $M$ be a set of functions in $L(0,1)$. If

$1^{\circ}$ there exists a constant $K$ such that for all $x(t) \in M$

$$
\int_{0}^{1}|x(t)| d t \leqq K
$$

and if

$2^{\circ}$ for every $\varepsilon>0$, there is $a \delta>0$ such that for all $x(t) \in M$ and all $h$ for which $|h|<\delta$

$$
\int_{0}^{1}|x(t+h)-x(t)| d t<\varepsilon,
$$

then the set $M$ is conditionally compact in the sense of the metric of L. A proof of the above result can be found in Nemyckii [1].

THEOREM B (Fubini converse: L. Tonelli). Let $f(x, y)$ be measurable 
on the unit square. If for almost all $x,|f(x, y)|$ is summable as a. function of $y$, and if

$$
\int_{0}^{1} d x \int_{0}^{1}|f(x, y)| d y
$$

exists as an iterated integral and is finite, then $f(x, y)$ is summable: on the unit square.

A proof of this theorem is in McShane [1].

1. Proof of theorem 1. Define $g(p, q)=f(p, q)$ for $p \neq q$ and $g(p, p)=0$. Then $g$ satisfies condition (C) and $f \leqq g$. Choose a fixed $s \in S$ and define

$$
\varphi(p)=\operatorname{lub}\left\{g\left(p, q_{1}\right)+g\left(q_{1}, q_{2}\right)+\cdots+g\left(q_{n-1}, q_{n}\right)+g\left(q_{n}, s\right)\right\}
$$

where the least upper bound is taken over all finite sequences $q_{1}, \cdots, q_{n}$ selected from $S$. Since $g$ satisfies (C) the finite sum is always $\leqq-g(s, p)$, and so the least upper bound is finite. Now fix $q_{1}=q$ and let the remaining $q_{i}$ range unrestricted. The definition of $\varphi$ yields at once $\varphi(p) \geqq g(p, q)+\varphi(q)$ so that $f(p, q) \leqq g(p, q) \leqq \varphi(p)-\varphi(q)$ as claimed. Finally $f(p, s) \leqq g(p, s) \leqq \varphi(p) \leqq-g(s, p) \leqq-f(s, p)$, which completes the proof.

It may be worth remarking that if the range of $f$ is any conditionally complete lattice ordered group, the proof goes through unchanged.

2. Proof of the matrix theorem. In this section we give a proof of inequality (1) based on Theorem 1. Suppose as stated in the introduction that the $n \times n$ matrix $A$ is doubly stochastic and that $\Pi a_{i i} \geqq$ $\Pi a_{i \sigma(i)}$ for all permutations $\sigma$. It is technically convenient to assume for the moment also that $a_{i j}>0$.

Let $b_{i j}=\log a_{i j}-\log a_{i i}$; then $b_{i j}$ as a function on $S \times S, S=$ $\{1,2, \cdots, n\}$, is easily seen to satisfy condition (C). (This follows readily from $b_{i i}=0$ and $\sum b_{i \sigma(i)} \leqq 0$ for all $\sigma$.) Hence there exists a vector $c_{i}$ such that $b_{i j} \leqq c_{i}-c_{j}$. Thus

$$
\log a_{i j} \leqq \log a_{i i}+c_{i}-c_{j}, \quad i, j=1, \cdots, n,
$$

so that

$$
a_{i j} \log a_{i j} \leqq a_{i j} \log a_{i i}+a_{i j} c_{i}-a_{i j} c_{j} .
$$

If we now sum first with respect to $j$ and then with respect to $i$, the vector $c_{i}$ drops out and we have

$$
\sum \sum a_{i j} \log a_{i j} \leqq \sum \log a_{i i} .
$$


The positivity restriction of the $a_{i j}$ is easily removed by a simple continuity argument.

3. Functions on the unit square. In this and the following section we shift our attention from the discrete matrix situation of $\S 2$ and study an analogous situation on the unit square.

Let $I$ denote the half open unit interval $[0,1)$ and $\mathscr{T}$ the class of one-to-one measure preserving transformations of $I$ onto $I$. We will prove the following theorem:

THEOREM 2. Let $f(x, y)$ be a measurable function on $I \times I$ which satisfies

and

$$
1^{\circ} \text { for all } T \in \mathscr{T}, f(x, T x) \in L(I) \text { and } \int_{0}^{1} f(x, T x) d x \leqq 0,
$$

$2^{\circ}$ the limit as $\delta \rightarrow 0$ of $\int_{0}^{1}|f(x, x+\delta)| d x=0$.

(The function $f(x, y)$ is defined outside $I \times I$ to be periodic of period one in $x$ and $y$.) Then there exists a function $\varphi \in L(I)$ such that for almost all $(x, y) \in I \times I$

$$
f(x, y) \leqq \varphi(x)-\varphi(y) .
$$

The proof of Theorem 2 requires two lemmas. (Throughout this section we will assume that $1^{\circ}$ and $2^{\circ}$ above hold.)

LEMma 1. Let $E \subset I$ be the union of a finite number of disjoint intervals and let $T \in \mathscr{T}$ be such that $T E=E$. Then

$$
\int_{E} f(x, T x) d x \leqq 0
$$

Proof. We may assume that the intervals of $E$ are semi-open (open on the right), so that the same is true of the finite set of noncontinuous intervals that compose $I-E$. Let $J=[a, b)$ be one such interval of $I-E$. Define a measure preserving transformation $U_{n}$ on $J$ as follows: set $\delta_{n}=(b-a) / 2 n$ and

$$
\begin{aligned}
& U_{n} x=x+\delta_{n}, a+2(k-1) \delta_{n} \leqq x<a+(2 k-1) \delta_{n} \\
& U_{n} x=x-\delta_{n}, a+(2 k-1) \delta_{n} \leqq x<a+2 k \delta_{n}, \\
& k=1, \cdots, n .
\end{aligned}
$$

Then

$$
\begin{aligned}
\left|\int_{J} f\left(x, U_{n} x\right) d x\right| & \leqq \int_{0}^{1}\left|f\left(x, x+\delta_{n}\right)\right| d x+\int_{0}^{1}\left|f\left(x, x-\delta_{n}\right)\right| d x \\
& \rightarrow 0 \text { as } n \rightarrow \infty \text { by } 2^{\circ} \text { of Theorem } 2 .
\end{aligned}
$$


If we define $U_{n}$ similarly on each of the finite set of $J \subset I-E$, and $U_{n} x=T x$ for $x \in E$, then $U_{n} \in \mathscr{T}$ and

$$
\int_{I-E} f\left(x, U_{n} x\right) d x+\int_{E} f(x, T x) d x \leqq 0,
$$

by $1^{\circ}$ of Theorem 2 . Since $\int_{I-E} f\left(x, U_{n} x\right) d x \rightarrow 0$, the result follows.

Lemma 2. Let $f(x, y)$ be as in Theorem 2. Define for $0<\lambda<1$

$$
f(x, y ; \lambda)=\frac{1}{\lambda} \int_{0}^{\lambda} f(x+t, y+t) d t .
$$

Then $f(x, y ; \lambda)$ satisfies condition (C) of Theorem 1 on $I \times I$.

Proof. We prove the lemma for the function $\lambda f(x, y ; \lambda)$. Define $F\left(x_{1}, x_{2}, \cdots, x_{n} ; \lambda\right)=\lambda f\left(x_{1}, x_{2} ; \lambda\right)+\cdots+\lambda f\left(x_{n}, x_{1} ; \lambda\right) \equiv F(x ; \lambda)$. We will show that given any ordered set $x=\left(x_{1}, \cdots, x_{n}\right), F(x ; \lambda) \leqq 0$ for all $0<\lambda<1$. The following two easily verified properties of $F(x ; \lambda)$ will be required:

(5a) given any finite ordered set $x$, there are finite ordered sets $x^{(i)}$, each of which has distinct components, and elements $x_{j}$, such that identically in $\lambda$

$$
\begin{aligned}
F(x ; \lambda)= & F\left(x^{(1)} ; \lambda\right)+\cdots+F\left(x^{(k)} ; \lambda\right) \\
& +F\left(x_{1}, x_{1} ; \lambda\right)+\cdots+F\left(x_{p}, x_{p} ; \lambda\right) ;
\end{aligned}
$$

(5b) identically in $x$

$$
F(x ; \lambda)=F\left(x ; \lambda_{1}\right)+F\left(x+\lambda_{1} ; \lambda_{2}\right)+\cdots+F\left(x+\lambda_{1}+\cdots+\lambda_{k-1} ; \lambda_{k}\right),
$$

where $\lambda=\lambda_{1}+\cdots+\lambda_{k}$.

(We leave to the reader the verification of the above.)

As a consequence of Lemma $1\left(F\left(x_{j}, x_{j} ; \lambda\right) \leqq 0\right)$ and (5a), it will suffice to prove $F(x ; \lambda) \leqq 0$ when the components of $x$ are distinct. Suppose then that $x=\left(x_{1}, \cdots, x_{n}\right), x_{i} \neq x_{j}$ for $i \neq j, 0 \leqq x_{i}<1$, and consider for the moment the $x_{i}$ rearranged in increasing order, say $y_{1}, \cdots, y_{n}$. We define $\lambda_{*}=\operatorname{Min}\left\{y_{2}-y_{1}, y_{3}-y_{2}, \cdots, y_{n}-y_{n-1}, y_{1}+\right.$ $\left.1-y_{n}\right\}$, and note that $\lambda_{*}>0$ by our conditions on the $x_{i}$. Suppose first that $0<\lambda \leqq \lambda_{*}$, and let $E$ be the set of points $x_{i}+t(i=1, \cdots, n$; $0 \leqq t<\lambda)$ reduced modulo 1 . For $0 \leqq t<\lambda$ define $T\left(x_{i}+t\right)=x_{i+1}+t$, $i=1, \cdots, n-1$ and $T\left(x_{n}+t\right)=x_{1}+t$, where again all numbers are reduced modulo 1 . Since $\lambda \leqq \lambda_{*}, T$ is well defined on $E$ and $T E=E$. For $x \in I-E$, define $T x=x$, and we have $T \in \mathscr{T}$. By the periodicity of $f$,

$$
F(x ; \lambda)=\int_{E} f(x, T x) d x \text {, which is } \leqq 0 \text { by Lemma } 1
$$


We have shown, then, that

$$
\text { for } 0<\lambda \leqq \lambda_{*}, \quad F(x ; \lambda) \leqq 0 .
$$

Finally, since for $0<\lambda<1$ we may write $\lambda=k \lambda_{*}+r$ where $k$ is: a nonnegative integer and $0 \leqq r<\lambda_{*}$, we see that (5b) and (6) complete: the proof. (This is equivalent to iterating $T k$ times with $\lambda=\lambda_{*}$ and then using $T$ with $\lambda=r$.)

Before staring the proof of Theorem 2 we make a heuristic remark about hypothesis $2^{\circ}$. If $f(x, y) \leqq \varphi(x)-\varphi(y), f(x, x)=0$, and all the functions are smoothly differentiable, then the surfaces $\mathrm{z}=f(x, y)$ and $z=\varphi(x)-\varphi(y)$ are tangent along $y=x$, and so $\varphi(x)$ is determined (up to an additive constant) by $\varphi^{\prime}(x)=f_{1}(x, x)$. This suggests strongly that the "nature" of $\varphi$ in general is determined by the behavior of $f(x, y)$ in the neighborhood of $y=x$. This will become clear in the proof that follows; later we will mention some consequences to $\varphi$ of altering $2^{\circ}$.

We proceed now to the proof of Theorem 2. By Theorem 1 and Lemma 2 we know that for each $\lambda, 0<\lambda<1$, and for any $s \in I$, we can find a function $\varphi(x ; s, \lambda)$ such that for all $(x, y) \in I \times I$

$$
\begin{aligned}
& f(x, y ; \lambda) \leqq \varphi(x ; s, \lambda)-\varphi(y ; s, \lambda), \\
& f(x, s ; \lambda) \leqq \varphi(x ; s, \lambda) \leqq-f(s, x ; \lambda),
\end{aligned}
$$

and

$$
\varphi(s ; s, \lambda)=0 .
$$

The remainder of the proof will be devoted to analyzing the (conditional) compactness of the family $\{\varphi(x ; s, \lambda)\}$ in $L(I)$.

Theorem A (Riesz-Arzela) tells us that conditional compactness is implied by equicontinuity and uniform boundedness. We have from (7)

$$
f(x, y ; \lambda) \leqq \varphi(x ; s, \lambda)-\varphi(y ; s, \lambda) \leqq-f(y, x ; \lambda),
$$

so that

$$
\begin{aligned}
|\varphi(x+\delta ; s, \lambda)-\varphi(x ; s, \lambda)| & \leqq|f(x+\delta, x ; \lambda)|+|f(x, x+\delta ; \lambda)| \\
& \leqq \frac{1}{\lambda} \int_{0}^{1}\{|f(x+\delta, x)|+|f(x, x+\delta)|\} d x .
\end{aligned}
$$

Thus by $2^{\circ}, \varphi(x ; s, \lambda)$ is continuous and hence measurable. Furthermore from the first inequality of (9) and Theorem $B$ we have easily

$$
\begin{aligned}
\int_{0}^{1} \mid \varphi(x+ & \delta ; s, \lambda)-\varphi(x ; s, \lambda) \mid d x \\
& \leqq \int_{0}^{1}\{|f(x+\delta, x)|+|f(x, x+\delta)|\} d x,
\end{aligned}
$$


so that the entire family $\{\varphi(x ; s, \lambda)\}$ is equicontinuous $(L)$.

Uniform boundedness $(L)$ is more of a problem. We have found it necessary to choose an appropriate sub-family, and this will be done in the following paragraphs.

Since $f(x, y)$ is measurable on $I \times I$ we conclude from $2^{\circ}$ and Theorem $B$ that there exists a number $a>0$ such that $f$ is summable on the set $P$ bounded by the lines $x=0, x=1, y=x \pm a$. We define $\bar{f}(x, y)=f(x, y)$ on $P$ and all points in the plane congruent to $P$ modulo one in $x$ and $y$; elsewhere we set $\bar{f}(x, y)=0$.

We will choose $s_{1} \in I$ so that $0 \leqq s_{1}<a$, and both (11) and (12) are satisfied:

$$
\begin{aligned}
& \text { as } \lambda \rightarrow 0 \\
& \lim \frac{1}{\lambda} \int_{s_{1}}^{s_{1}+\lambda} d x \int_{0}^{1}|\bar{f}(x, y)| d y=\int_{0}^{1}\left|\bar{f}\left(s_{1}, y\right)\right| d y<\infty,
\end{aligned}
$$

and

$$
\lim \frac{1}{\lambda} \int_{s_{1}}^{s_{1}+\lambda} d y \int_{0}^{1}|\bar{f}(x, y)| d x=\int_{0}^{1}\left|\bar{f}\left(x, s_{1}\right)\right| d x<\infty
$$

and

$$
\begin{gathered}
\text { as } n \rightarrow \infty, \text { for almost all } x \in I, \\
\lim f_{n}\left(s_{1}, x\right)=f\left(s_{1}, x\right), \text { and } \lim f_{n}\left(x, s_{1}\right)=f\left(x, s_{1}\right),
\end{gathered}
$$$$
\text { where } f_{n}(x, y) \equiv f\left(x, y ; n^{-1}\right) \text {. }
$$

For almost all $s \in I$ (11) holds since $\bar{f} \in L(P)$ and so $\in L(I \times I)$. Similarly, (12) is valid for almost all $s \in I$ by the fundamental theorem of calculus. (We introduce $f_{n}$ in (12) to avoid some possible measurability difficulties.) Thus $s_{1}$ can certainly be chosen as required.

We will now show that the family $\left\{\varphi\left(x ; s_{1}, n^{-1}\right)\right\}$ is uniformly bounded $(L)$. We choose $s_{2}, \cdots, s_{k}$ so that

$$
\begin{gathered}
s_{1}<s_{2}<\cdots<s_{k}<1, \\
s_{i+1}-s_{i}<2 a \text { for } i=1, \cdots, k-1, \text { and } 1-s_{k}<a ;
\end{gathered}
$$

(14) $s_{i}$ satisfies (11) when $s_{1}$ is replaced by $s_{i}, i=2, \cdots, k$; and finally

$$
\begin{aligned}
& \text { as } n \rightarrow \infty, \\
& \lim f_{n}\left(s_{1}, s_{i}\right)=f\left(s_{1}, s_{i}\right),
\end{aligned}
$$

and

$$
\lim f_{n}\left(s_{i}, s_{1}\right)=f\left(s_{i}, s_{1}\right), \quad i=2, \cdots, k .
$$

Now define $\left[a_{1}, b_{1}\right)=\left[0, s_{1}+a\right),\left(a_{k}, b_{k}\right)=\left(s_{k}-a, 1\right)$, and $\left(a_{i}, b_{i}\right)=$ 
$\left(s_{i}-a, s_{i}+a\right), i=2, \cdots, k-1$. The union of these intervals covers

I. Write $\varphi_{n}\left(x ; s_{1}\right)$ for $\varphi\left(x ; s_{1}, n^{-1}\right)$. Then by (8)

$$
\begin{gathered}
\left|\varphi_{n}\left(x ; s_{1}\right)\right| \leqq\left|f_{n}\left(x, s_{i}\right)\right|+\left|f_{n}\left(s_{i}, x\right)\right|+\left|\varphi_{n}\left(s_{i} ; s_{1}\right)\right|, \\
\text { for } x \in I \text { and } i=1, \cdots, k .
\end{gathered}
$$

Hence

$$
\begin{aligned}
& \int_{a_{i}}^{b_{i}}\left|\varphi_{n}\left(x ; s_{1}\right)\right| d x \\
& \quad \leqq \int_{a_{i}}^{b_{i}}\left|f_{n}\left(x, s_{i}\right)\right| d x+\int_{a_{i}}^{b_{i}}\left|f_{n}\left(s_{i}, x\right)\right| d x+\left(b_{i}-a_{i}\right)\left|\varphi_{n}\left(s_{i}, s_{1}\right)\right|, \\
& \quad \leqq A_{i}+B_{i}+C_{i}, \quad \text { where, }
\end{aligned}
$$

for $1 \leqq i \leqq k$, by $(14)$

$$
\begin{aligned}
& A_{i}=\operatorname{lub}\left\{n \int_{s_{i}}^{s_{i}+n^{-1}} d y \int_{0}^{1}|\bar{f}(x, y)| d x\right\}<\infty, \\
& B_{i}=\operatorname{lub}\left\{n \int_{s_{i}}^{s_{i}+n^{-1}} d x \int_{0}^{1}|\bar{f}(x, y)| d y\right\}<\infty ; \\
& C_{1}=0, \text { by (7), }
\end{aligned}
$$

and for $2 \leqq i \leqq k$, by (15)

$$
C_{i}=\left(b_{i}-a_{i}\right) \operatorname{lub}\left\{\left|f_{n}\left(s_{i}, s_{1}\right)\right|+\left|f_{n}\left(s_{1}, s_{i}\right)\right|\right\}<\infty .
$$

Since

$$
\int_{0}^{1}\left|\varphi_{n}\left(x ; s_{1}\right)\right| d x \leqq \Sigma \int_{a_{i}}^{b_{i}}\left|\varphi_{n}\left(x ; s_{1}\right)\right| d x,
$$

we have established uniform boundedness $(L)$ and Theorem $\mathrm{A}$ applies. We have then that some subsequence $\left\{\varphi_{n_{i}}\left(x ; s_{1}\right)\right\}$ converges to $\varphi(x)$ (say) in $L$ and $f_{n_{i}}(x, y)$ converges to $f(x, y)$ for almost all $(x, y) \in I \times I$. Since for all $(x, y), f_{n}(x, y) \leqq \varphi_{n}\left(x ; s_{1}\right)-\varphi_{n}\left(y ; s_{1}\right)$, Theorem 2 follows.

We now return to our remark preceding the proof of the theorem. We have just seen that the fact that $\varphi$ is in $L(I)$ has been determined by condition $2^{\circ}$. It is reasonable to expect that a strengthening of $2^{\circ}$ should lead to a "smoothing" of $\varphi$, and this is indeed the case. If $2^{\circ}$ is replaced by

“ $2_{p}^{\circ}$ for fixed $p(1 \leqq p<\infty)$ the limit as $\delta \rightarrow 0$ of

$$
\int_{0}^{1}|f(x, x+\delta)|^{p} d x=0 "
$$

then $\varphi \in L_{p}(I)$. The modification of the proof consists of invoking the $L_{p}$ version of Theorem A, which is also to be found in Nemyckii [1]. Finally if we replace $2^{\circ}$ by 
" $2_{\infty}^{\circ}$ the limit as $\delta \rightarrow 0$ of ess $\sup _{x}|f(x, x+\delta)|=0$ ",

then $\varphi \in C(I)$. (The classical Arzela or Ascoli theorem is used.)

4. The permanent theorem in $L(I \times I)$. In this section we state and prove the $L(I \times I)$ analog of the discrete theorem of $\S 2$.

THEOREm 3. Suppose that $f(x, y)$ defined and measurable on $I \times I$ has the following properties:

$$
\begin{gathered}
1^{\circ} f(x, y)>0 \text { and } \int_{0}^{1} f(x, y) d x=\int_{0}^{1} f(x, y) d y=1, \text { for all } x, y ; \\
2^{\circ} \text { for all } T \in \mathscr{T}, f(x, T x) \text { is measurable, } \\
\qquad \log \frac{f(x, T x)}{f(x, x)} \in L(I),
\end{gathered}
$$

and

$$
\int_{0}^{1} \log \frac{f(x, T x)}{f(x, x)} d x \leqq 0
$$

and

$$
3^{\circ} \quad \int_{0}^{1}\left|\log \frac{f(x, x+\delta)}{f(x, x)}\right| d x \rightarrow 0, \quad \text { as } \delta \rightarrow 0 .
$$

Then $f \log f \in L(I \times I)$ and

$$
\infty>\int_{0}^{1} \log f(x, x) d x \geqq \int_{0}^{1} \int_{0}^{1} f(x, y) \log f(x, y) d x d y \geqq 0 .
$$

Proof. Conditions $2^{\circ}$ and $3^{\circ}$ above suffice for the application of Theorem 2 to the function $\log [f(x, y) / f(x, x)]$ : there exists $\varphi(x) \in L(I)$ such that for almost all $x, y$

$$
\log \frac{f(x, y)}{f(x, x)} \leqq \varphi(x)-\varphi(y) .
$$

If we multiply by $f(x, y)$ and rearrange, we find

$$
\begin{aligned}
-\frac{1}{e} & \leqq f(x, y) \log f(x, y) \\
& \leqq f(x, y) \log f(x, x)+\varphi(x) f(x, y)-\varphi(y) f(x, y),
\end{aligned}
$$

where the first inequality is a consequence of $-1 / e=g l b x \log x$ for $x>0$. Now, as functions of $y, f(x, y) \log f(x, x)$ and $\varphi(x) f(x, y)$ both $\in L$ by $1^{\circ}$ above. Again, if we apply Theorem B to $\varphi(y) f(x, y)$, integrating first with respect to $x$, we see that $\varphi(y) f(x, y) \in L(I \times I)$, 
and so for almost all $x$, that function is summable as a function of $y$. Thus by (21), $f(x, y) \log f(x, y)$ is summable $y$ for almost all $x$, and integrating gives

$$
\begin{aligned}
0 & \leqq \int_{0}^{1} f(x, y) \log f(x, y) d y \\
& \leqq \log f(x, x)+\varphi(x)-\int_{0}^{1} \varphi(y) f(x, y) d y .
\end{aligned}
$$

(The first inequality above is Jensen: $\psi\left(\int_{0}^{1} f d y\right) \leqq \int_{0}^{1} \psi(f) d y$, where $\psi(x)=x \log x$.) Hence

$$
\log f(x, x) \geqq \int_{0}^{1} \varphi(y) f(x, y) d y-\varphi(x),
$$

and so $\log f(x, x)$ is bounded below by a summable function. Now, since by $1^{\circ}$ and Theorem B $f \in L(I \times I)$, it follows that for almost all $\delta, f(x, x+\delta) \in L(I)$. We choose $\delta$ so that $f(x, x+\delta) \in L(I)$. Since $\log f(x, x+\delta)<f(x, x+\delta)$, and since by $2^{\circ} \log f(x, x+\delta)$ $\log f(x, x) \in L(I)$, we see that

$$
\log f(x, x) \leqq f(x, x+\check{o})+\log [f(x, x) / f(x, x+\delta)],
$$

and so $\log f(x, x)$ is also bounded above by a summable function; hence $\log f(x, x) \in L(I)$. Returning to (21) we apply Theorem B and have $f \log f \in L(I \times I)$; then integrating both sides of (22) $\varphi$ drops out and we have (19) as asserted.

\section{REFERENCES}

1. S. N. Afriat, [1] The system of inequalities $a_{r s}>X_{r}-X_{s}$, Proc. Camb. Phil. Soc., 59 (1963), 125-133.

2. G. Birkhoff, [1] Tres observaciones sobre el algebra lineal, Univ. Nac. Tucumán Rev. Ser. A5 (1946), 147-151.

3. M. Marcus, and H. Minc, [1] Some results on doubly stochastic matrices, Proc. Amer. Math. Soc., 13 (1962), 571-579.

4. M. Marcus, and M. Newman, [1] On the minimum of the permanent of a doubly stochastic matrix, Duke Math. J., 26 (1959), 61-72.-[2] The permanent function as an inner product, Bull. Amer. Math. Soc., 67 (1961), 223-224.

5. E. J. Mc Shane, [1] Integration, Princeton Univ. Press, Princeton, New Jersey (1947).

6. V. V. Nemyckii, [1] The method of fixed points in analysis, Uspekhi Mat. Nauk., 1 (1936), 141-174.

Sandia laboratory, Albuquerque, New Mexico 


\section{PACIFIC JOURNAL OF MATHEMATICS}

\section{EDITORS}

\author{
Robert Osserman \\ Stanford University \\ Stanford, California
M. G. Arsove
University of Washington
Seattle 5 , Washington

\author{
J. DugundJI \\ University of Southern California \\ Los Angeles 7, California
}

Lowell J. Paige

University of California

Los Angeles 24, California

\section{ASSOCIATE EDITORS}
E. F. BECKENBACH
B. H. NeumanN
F. WOLF
K. YOSIDA

\section{SUPPORTING INSTITUTIONS}

\author{
UNIVERSITY OF BRITISH COLUMBIA \\ CALIFORNIA INSTITUTE OF TECHNOLOGY \\ UNIVERSITY OF CALIFORNIA \\ MONTANA STATE UNIVERSITY \\ UNIVERSITY OF NEVADA \\ NEW MEXICO STATE UNIVERSITY \\ OREGON STATE UNIVERSITY \\ UNIVERSITY OF OREGON \\ OSAKA UNIVERSITY \\ UNIVERSITY OF SOUTHERN CALIFORNIA
}

\author{
STANFORD UNIVERSITY \\ UNIVERSITY OF TOKYO \\ UNIVERSITY OF UTAH \\ WASHINGTON STATE UNIVERSITY \\ UNIVERSITY OF WASHINGTON \\ * * * * \\ AMERICAN MATHEMATICAL SOCIETY \\ CALIFORNIA RESEARCH CORPORATION \\ SPACE TECHNOLOGY LABORATORIES \\ NAVAL ORDNANCE TEST STATION
}

Mathematical papers intended for publication in the Pacific Journal of Mathematics should by typewritten (double spaced), and on submission, must be accompanied by a separate author's résumé. Manuscripts may be sent to any one of the four editors. All other communications to the editors should be addressed to the managing editor, L. J. Paige at the University of California, Los Angeles 24, California.

50 reprints per author of each article are furnished free of charge; additional copies may be obtained at cost in multiples of 50 .

The Pacific Journal of Mathematics is published quarterly, in March, June, September, and December. Effective with Volume 13 the price per volume (4 numbers) is $\$ 18.00$; single issues, $\$ 5.00$. Special price for current issues to individual faculty members of supporting institutions and to individual members of the American Mathematical Society: $\$ 8.00$ per volume; single issues $\$ 2.50$. Back numbers are available.

Subscriptions, orders for back numbers, and changes of address should be sent to Pacific Journal of Mathematics, 103 Highland Boulevard, Berkeley 8, California.

Printed at Kokusai Bunken Insatsusha (International Academic Printing Co., Ltd.), No. 6, 2-chome, Fujimi-cho, Chiyoda-ku, Tokyo, Japan.

PUBLISHED BY PACIFIC JOURNAL OF MATHEMATICS, A NON-PROFIT CORPORATION

The Supporting Institutions listed above contribute to the cost of publication of this Journal, but they are not owners or publishers and have no responsibility for its content or policies. 


\section{Pacific Journal of Mathematics}

\section{Vol. 14, No. $3 \quad$ July, 1964}

Erik Balslev and Theodore William Gamelin, The essential spectrum of a class of ordinary differential operators . . . . . . . . . . . . . . . . . . . .

James Henry Bramble and Lawrence Edward Payne, Bounds for derivatives in

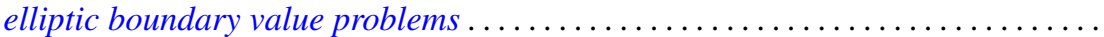

Hugh D. Brunk, Integral inequalities for functions with nondecreasing

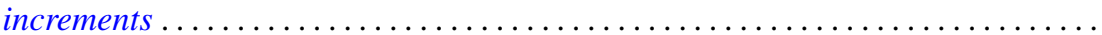

William Edward Christilles, A result concerning integral binary quadratic

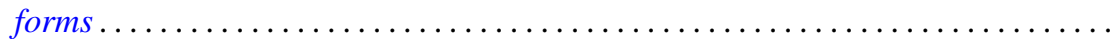

Peter Crawley and Bjarni Jónsson, Refinements for infinite direct decompositions of

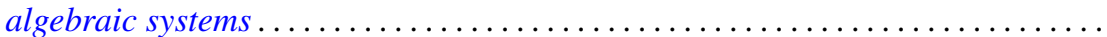

Don Deckard and Carl Mark Pearcy, On continuous matrix-valued functions on a Stonian space.

Raymond Frank Dickman, Leonard Rubin and P. M. Swingle, Another

characterization of the $n$-sphere and related results $\ldots \ldots \ldots \ldots \ldots \ldots$

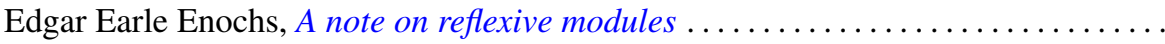

Vladimir Filippenko, On the reflection of harmonic functions and of solutions of the

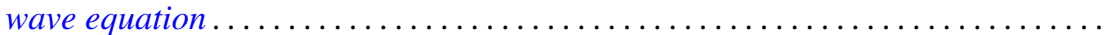

Derek Joseph Haggard Fuller, Mappings of bounded characteristic into arbitrary

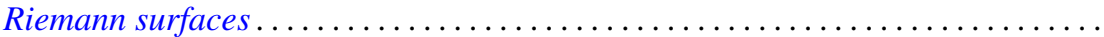
895

Curtis M. Fulton, Clifford vectors . . . . . . . . . . . . . . . . . . . . . . . . . . . . . . 917

Irving Leonard Glicksberg, Maximal algebras and a theorem of Radó . .

919

Kyong Taik Hahn, Minimum problems of Plateau type in the Bergman metric

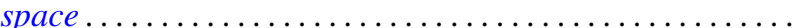

A. Hayes, A representation theory for a class of partially ordered rings...

J. M. C. Joshi, On a generalized Stieltjes trasform

J. M. C. Joshi, Inversion and representation theorems for a generalized Laplace transform ...

Eugene Kay McLachlan, Extremal elements of the convex cone $B_{n}$ of functions ...

Robert Alan Melter, Contributions to Boolean geometry of p-rings ...

James Ronald Retherford, Basic sequences and the Paley-Wiener criterion . . . . . . . 1019

Dallas W. Sasser, Quasi-positive operators. .

Oved Shisha, On the structure of infrapolynomials with prescribed coefficients ..

Oved Shisha and Gerald Thomas Cargo, On comparable means

Maurice Sion, A characterization of weak ${ }^{*}$ convergence ........

Morton Lincoln Slater and Robert James Thompson, A permanent inequality for

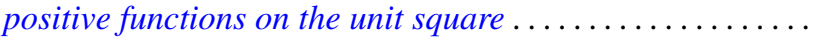

David A. Smith, On fixed points of automorphisms of classical Lie algebras ...

Sherman K. Stein, Homogeneous quasigroups ................

J. L. Walsh and Oved Shisha, On the location of the zeros of some infrapolynomials with prescribed coefficients .

Ronson Joseph Warne, Homomorphisms of $d$-simple inverse semigroups with identity . 